\title{
Eludication of the centromere involvement in an inversion (13) by fluorescent in situ hybridisation
}

Patricia L Gordon, James D Dalton, Paula R Martens, Avirachan T Tharapel, R Sid Wilroy

\begin{abstract}
A newborn infant with phenotypic features of trisomy for distal 13q was found to have recombinant inversion duplication involving the (13)(q22 $\rightarrow$ qter) region. Parental karyotypes showed that the mother had a normal $46, X X$ complement and the father had an apparently balanced pericentric inversion of a chromosome 13. Because of the unusual nature of the inversion, the exact position of the centromere on the father's inverted chromosome 13 was difficult to assign by GTG banding, even on prometaphase chromosomes. CBG and NOR banding were not informative in determining the location of the centromere. Fluorescent in situ hybridisation with an $\alpha$ satellite DNA probe for D13Z1/D21Z1 helped in confirming the exact position of the centromere in the rearranged paternal chromosome. Thus, the origin of the proband's abnormal chromosome 13 was clarified.
\end{abstract}

(f Med Genet 1993;30:414-16)

Partial trisomy for the proximal and distal regions of chromosome 13 constitute two distinct clinical entities. ${ }^{1-4}$ Partial duplications of distal 13 (q21 or $22 \rightarrow$ qter) are known to result from familial balanced pericentric inversions of chromosome 13..$^{5-8}$ The mechanism leading to the duplication of a segment in an inversion heterozygote is crossing over within the inversion loop during meiosis. In this report we describe a patient with partial duplication of chromosome 13 (q22 $\rightarrow$ qter) and the use of fluorescent in situ hybridisation (FISH) with an $\alpha$ satellite DNA probe to delineate a balanced pericentric inversion 13(p11.2q22) in her father. $\alpha$ satellite DNA is composed of heterogeneous repeated sequences which are unique to most human chromosomes. Exceptions such as the $13 / 21 \alpha$ satellite probe that hybridises to the centromeres of more than one chromosome pair are known. Yet even in these cases, the size differences of chromosomes 12 and 21 would allow the detection of the presence and location of centromeres. In the family presented here, we used a 13/21 $\alpha$ satellite probe for FISH which allowed the confirmation of the centromere position on the paternal inverted 13 .

\section{Case report}

The proband was referred for genetic evaluation at 3 days of age because of dysmorphic features and multiple congenital anomalies. She was born to an 18 year old mother and a 24 year old father. Both parents were in good health at the time of the proband's conception. Labour was induced because of post-term status and meconium stained amniotic fluid. Birth weight was $3745 \mathrm{~g}$ (90th centile) and length was $51.5 \mathrm{~cm}$ ( 75 th centile). Respiratory distress occurred shortly after birth and a congenital heart defect was suspected. However, cardiac evaluation showed no anomalies. Review of the family history indicated that the maternal grandmother had one spontaneous fetal loss at six months' gestation. Dysmorphic physical features of the proband included the following: flat occiput, large anterior fontanelle, open sagittal suture, capillary haemangiomas of the face, forehead, nasal bridge, eyelids, upper lip, and back, colobomas of the irides, left microphthalmos, sparse eyelashes and eyebrows, low set, large ears with broadly rolled helices, transverse nasal crease, long, bulbous nose, micrognathia, short neck, prominent sternum, narrow thorax, prominent labia majora, hirsutism of the lower back, pilonidal dimple, rocker bottom feet, postaxial hexadactyly of the hands and feet, and long, narrow fingers. She was hypertonic with incomplete extension of the hips, knees, and elbows. Renal ultrasonogram showed enlarged kidneys with duplication of the collecting system on the left. Further ophthalmological evaluation showed left optic nerve coloboma with left optic nerve agenesis.

\section{CYTOGENETICS}

Chromosome analysis using conventional GTG banding showed a recombinant inversion duplication of the distal $13 q$ region with a $46, \mathrm{XX}$, rec(13), inv dup (q22 $\rightarrow$ qter) complement (fig 1A). Parental karyotypes showed the mother had a normal 46,XX complement and 
A
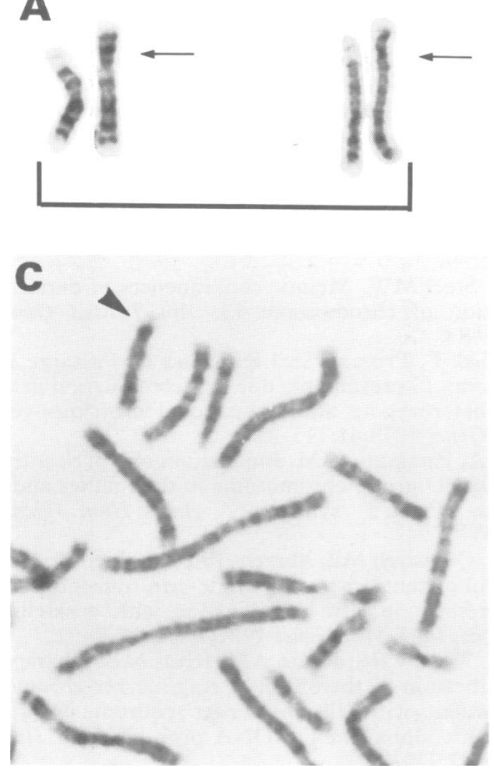

Figure 1 (A) Partial karyotype of the proband's normal and abnormal chromosome 13 with inversion duplication. (B) Partial karyotype of proband's father's normal and inverted chromosome 13. (C, D) A partial metaphase from the father stained with GTG and NOR banding, respectively. Note the NOR positive area at the terminus of the inverted 13.
$\mathbf{B}$

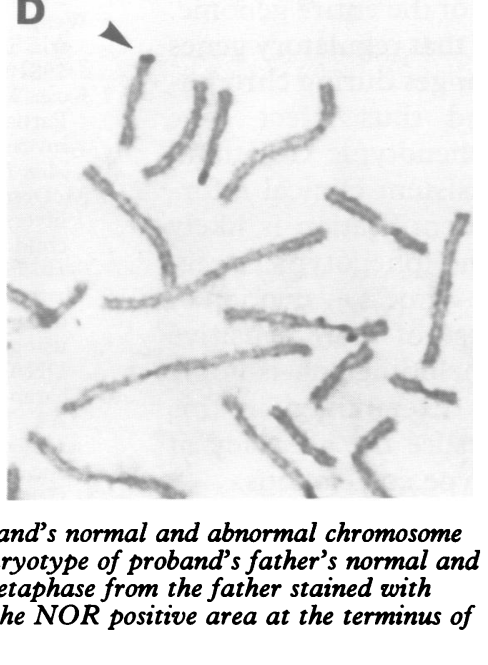

the father had an apparently balanced pericentric inversion of one chromosome 13 (fig 1B). The inverted 13 had a satellite and satellite stalk at one terminus. This made the localisation of the centromere all the more difficult despite GTG, CBG, and NOR banding analyses. A partial metaphase spread stained by GTG and NOR banding showing the inverted and normal chromosome $13 \mathrm{~s}$ of the father is shown in fig $1 \mathrm{C}$ and D. FISH with an $\alpha$ satellite DNA probe for D13Z1/D21Z1 was useful in resolving the position of the centromere in the rearranged chromosome 13 of the father. The inversion was interpreted as pericentric with a 46,XY,inv(13)(p1lq22) (fig 2). The probe for $\mathrm{D} 13 \mathrm{Z1} / \mathrm{D} 21 \mathrm{Z1}$ was obtained commercially (Oncor Inc, Gaithersburg, MD) and was used according to the manufacturer's instructions. The hybridisation was detected

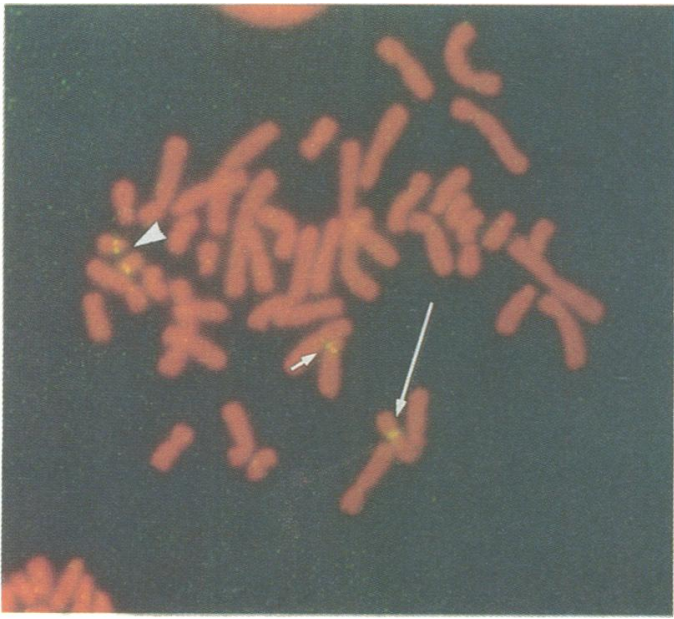

Figure 2 Fluorescent in situ hybridisation with the probe for D13Z1/D21Z1. Long arrow shows the normal chromosome 13 and short arrow shows the inverted chromosome 13 with the centromere located in the middle of the chromosome. Arrowhead shows the two chromosome 21 s with cross hybridisation. by FITC labelled avidin, signals were amplified by antiavidin antibody, and chromosomal DNA was counterstained by propidium iodide. The slides were viewed and photographed under a Zeiss Fluorescence Photomicrocope II using Kodacolor Gold 200 film.

\section{Discussion}

A patient with a recombinant inversion duplication resulting in partial trisomy 13 led to the identification of a parent with a pericentric inversion of chromosome 13 . The inversion duplication of (13)(q21 or $22 \rightarrow$ qter) usually originates through synaptic crossing over within the inversion loop in an inv(13)(p11q21) heterozygote. Morphologically, the inverted chromosome may rarely appear as an acrocentric itself ${ }^{5}$ or may appear as metacentric. ${ }^{6}$ Using the conventional chromosome banding techniques the centromere position in the inverted chromosome may be difficult to determine precisely. The GTG banded chromosome 13 in the parent of our patient had an acrocentric appearance with a satellite on one terminus (fig 1C). On NOR banding silver stain positivity was seen at the same terminus of the inverted chromosome (fig 1D). A clear definition of the centromere and the pericentric nature of the inversion in the father was possible only through FISH with a 13/21 $\alpha$ satellite DNA probe (fig 2).

The use of FISH to determine the chromosomal origin of the centromeres in whole arm translocations and to define the origin of ring and marker chromosomes has been reported previously. ${ }^{910}$ When dealing with certain pericentric inversions, it may be difficult to determine the location of the centromere. This can lead to questionable cytogenetic interpretations. Accuracy in determining the area involved is important in many cases, such as partial trisomy $13 \mathrm{q}$ in which phenotypic variability occurs. The delineation of the mechanisms responsible for this variability can only be enhanced by more consistent nomenclature and by more accurate clinical descriptions.

From a clinical standpoint, it is important to address the fact that most of the features seen in our proband are consistent with trisomy for the distal portion of 13q. In distal 13q partial trisomy, the most consistent features noted in published reports are polydactyly, haemangiomas, high arched palate, and cryptorchidism. ${ }^{2}$ The latter does not apply as our patient is female. Polydactyly and haemangiomas were present but high arched palate was not. Polydactyly has been associated with trisomy of $13 \mathrm{q} 31$ and haemangiomas with trisomy of $13 q 32 \rightarrow$ qter. Both features are consistent with the inversion duplication of $13 \mathrm{q} 22 \rightarrow \mathrm{qter}$ in our patient. Noel et $a l^{4}$ suggested that the critical factor in the development of colobomas and microphthalmos is the presence of trisomy for the $13 \mathrm{q} 14.2$ or q14.3 regions. These regions are not present in triplicate in our patient who had colobomas and microphthalmos. Also, our patient had sparse eyebrows and eyelashes, which is in contrast to the review of Bonioli et $a l^{11}$ of 33 patients with partial $13 \mathrm{q}$ 
trisomy, all of whom had long, upward curved lashes. ${ }^{11}$

Rogers ${ }^{2}$ has previously addressed the phenotypic variation in cases of partial $13 q$ trisomy and discussed several explanations including that by Martin-Lucas et al ${ }^{12}$ suggesting that the expression of a chromosome abnormality may be under the control of the entire genome. It has also been proposed that regulatory genes may undergo position changes during chromosome rearrangement and thus affect gene expression resulting in phenotypic variation. ${ }^{2}$ $\mathrm{He}$ also noted that inconsistent clinical information and breakpoint identification is likely to be responsible for some 'phenotypic variation'. Detailed systematic clinical evaluation of these patients and the use of more sensitive cytogenetic techniques, including non-isotopic in situ hybridisation, as exemplified in this report, would further ensure the accuracy of future phenotype-karyotype correlations.

1 Tharapel SA, Lewandowski RC, Tharapel AT, Wilroy RS. Phenotype-karyotype correlation in patients trisomic fo various segments of chromosome 13. F Med Gene 1986;23:310-15.

2 Rogers JF. Clinical delineation of proximal and distal 13q trisomy. Clin Genet 1984;25:221-9.
3 Wilroy RS, Summitt RL, Martens P, Gooch WM. Partial monosomy and partial trisomy for different segments of chromosome 13 in several individuals of the same family. Ann Genet (Paris) 1977;20:237-42.

4 Noel B, Quack B, Rethoré MO. Partial deletions and trisomies of chromosome 13; mapping of bands associated with particular malformations. Clin Genet 1976;9:593602 .

5 Habedank M. Familial pericentric inversion of chromosome 13 resulting in duplication $13 \mathrm{q} 22 \rightarrow$ qter. $f$ Med Genet 1982;19:227-9.

6 Wenger SL, Steel MW. Meiotic consequences of pericentric inversion of chromosome 13. Am $\mathcal{J}$ Med Genet 1981;9:275-83.

7 Kose-Westphal T, Pruszak-Seel RE, Niss R, Passarge E. Partial trisomy 13 presumably due to recombination in an inversion heterozygote and by unequal crossing-over. Ann Hum Genet 1978;41:315-22.

$8 \mathrm{McDermott} A$, Parrington JM. Elucidation of a pericentric inversion of a $\mathrm{D}$ group chromosome in the mother and a child with Patau's syndrome. Ann Hum Genet child with Pat

9 Tharapel AT, Qumsiyeh MB, Martens PR, et al. Identification of the origin of centromeres in whole-arm translocations using fluorescent in situ hybridization with $\alpha$ satellite DNA probe. Am $\mathcal{f}$ Med Genet 1991;40:117-20.

10 Tharapel SA, Wilroy RS, Keath AM, Rivas ML, Tharapel AT. Identification of the origin of ring/marker chromosomes in patients with Ullrich-Turner syndrome using $X$ and $\mathrm{Y}$ specific alpha satellite DNA probes. Am $\mathcal{f} \mathrm{Hum}$ and Y specific alpha

11 Bonioli E, Crisalli M, Monteverde R, Vianello MG. Karyotype-phenotype correlation in partial trisomy $13 . \mathrm{Am} \mathcal{f}$ Dis Child 1981;135:1115-7.

12 Martin-Lucas MA, Perez-Castillo A, Abrisqueta JA, et al. Partial trisomy 13 due to maternal translocation t(7;13)(p22;q14). Ann Genet (Paris) 1982;25:172-8. 